\title{
Pulsar Signal Characteristic Frequency Identification And Phase Estimation Base On FFT
}

\author{
Hou Tianrui \\ dept. Research Center of Satellite Technology in HIT \\ name of organization, acronyms acceptable \\ Harbin, China \\ : e-mail: dzxxgch@sina.cn
}

\section{Xu Guodong}

dept. dept. Research Center of Satellite Technology in HIT

name of organization, acronyms acceptable

: Harbin, China

:xuguodong@163.com

\author{
Li Pengfei \\ dept. Research Center of Satellite Technology in HIT \\ : name of organization, acronyms acceptable \\ Harbin, China \\ lpf_365@163.com
}

Jialin.Hou

name of organization, acronyms acceptable

: Harbin, China

\begin{abstract}
To meet the detection of pulsars with low SNR, this paper presents a pulse signal recognition algorithm based on FFT. The new method could reduce the accumulation of time domain compared with the traditional method and could complete the detecting within a very short period of time First combined with the characteristics of pulsar signals ,make a theoretical description, for characteristic frequency identification method Second , after fft ,by looking at the peak of a specific frequency ,find the characteristic frequency, use it to estimate pulsar phase change in time domain .Third, Give the CRB(Cramer-Rao performance limit ) of characteristic frequency identification method. Use Mento-Carlo simulation for the simulation of pulsar PSRJ0007+7303. Simulation results show statistical results compared to the CRB in the same order of magnitude. Characteristic frequency identification method could achieve effective detection. The result shows Characteristic frequency identification method is an effective way for pulsar Identification and the evaluation of phase change which is directly related to the TOA parameters evaluation.
\end{abstract} Carlo

Keywords--pulsar; fft; phase evaluation ;CRB; Mento-

\section{INTRODUCTION}

Pulsar is a kind of high-speed rotating neutron stars. Neutron star is a continuous and stable celestial object radiating electromagnetic energy. The energy in the pole direction of the radiation intensity is large, and the energy in the other direction of the radiation intensity is small.

Therefore, in the course of neutron stars rotate, the observed signal from Earth shows cyclical changes.. Due to the stability of the neutron star spin, periodic pulse signal is also very stable. The period of pulse signal is as stable as the atomic clocks on Earth
For the periodic stability of the pulsar signal, using pul -sar to conduct aerospace navigation has became a focus of science study.

Now scientists have found about 1790 pulsars and there are about a few millisecond x-ray pulsars which is more available to be applied to deep space navigation. In table 1, we have given some millisecond pulsar characteristic parameters. Fig .1 is plot of X-ray sources from XNAVSC in Galactic longitude and latitude

\begin{tabular}{|} 
Table 1. Millisecond pulsar characteristic parameters \\
\begin{tabular}{|ccccc|}
\hline Name & $\mathbf{P}(\mathbf{m s})$ & $\begin{array}{c}\text { Flux } \\
\text { ph/(cm2 } \cdot \mathbf{s})\end{array}$ & $\begin{array}{c}\text { Pulsed } \\
\text { Fraction }\end{array}$ & $\begin{array}{c}\text { Duty } \\
\text { Cycle }\end{array}$ \\
\hline $\mathrm{B} 1937+21$ & 1.55 & 0.0000507 & 0.9 & 0.05 \\
\hline $\mathrm{B} 1821-24$ & 3.05 & 0.0000468 & 0.9 & 0.026 \\
\hline $\mathrm{J} 0218+4232$ & 2.32 & 0.0000383 & 0.69 & 0.11 \\
\hline $\mathrm{J} 1012+5307$ & 5.26 & 0.0000092 & 0.77 & 0.09 \\
\hline $\mathrm{J} 0437-4715$ & 5.75 & 0.0000079 & 0.4 & 0.35 \\
\hline $\mathrm{J} 2124-3358$ & 4.93 & 0.0000046 & 0.56 & 0.40 \\
\hline $\mathrm{J} 0030+0451$ & 4.86 & 0.0000038 & 0.69 & 0.15 \\
\hline $\mathrm{J} 0751+1807$ & 3.48 & 0.0000038 & 0.52 & 0.31 \\
\hline $\mathrm{J} 1024-0719$ & 5.16 & 0.0000004 & 0.52 & 0.40 \\
\hline
\end{tabular}
\end{tabular}

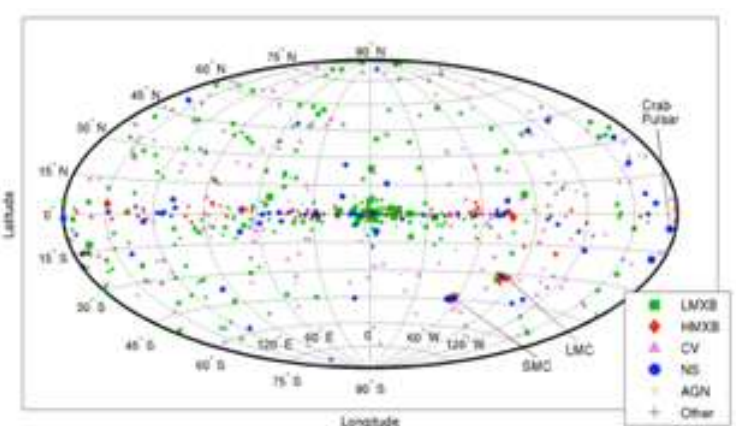

Figure 1.plotof X-ray sources from XNAVSC in Galactic longitude and latitude 
Now, Pulsar navigation is needed to address two important issues

1) Identify the pulsar signal

2) Measurements of Pulsar signal time of arrival (TOA)

As the TOA is directly related to the phase change of pulsar, it is equally to the measurements of the phase change.

The common approach is to accumulate the low SNR signal by huge pulsar detector for a long time and get the profile of pulsar .Then the obtained signal with the template in the standard library of pulsar profile to achieve identification. But this way cannot satisfy the real-time requirements of pulsar navigation. It is only a theory exploration and cannot be applied to astronomy engineer

To solve the above problem, this paper presents an FFT-based algorithm for the rapid detection of pulsars. Through the pulsar signal preprocessing, this algorithm can be effective in the noise suppression. In the Part One of the article, we will give the theoretical proof. Introduce the possibility and advantage of the algorithm basing in FFT. In the Part Two, the CRB theory will be introduced and the article gives the estimation formula for the algorithm. In the Part Three, the paper will give the simulation result. In the Part Four, the paper will analysis the results of simulation and draw the conclusions.

\section{PULSAR CHARACTERISTIC FREQUENCY IDENTIFICATION ALGORITHM}

Since each pulsar has a unique signal cycle, the pulsar cycle estimate is important for the feature recognition. The cycle is the Id for the pulsar.

\section{A. characteristic frequency}

Although pulsar signals are not generally sinusoidal signal, periodic signal can be decomposed into a series of sinusoidal signals. And the cycle of the pulsar signal is also the period of sinusoidal signal baseband signal. The phase change of pulsar is the same to the sinusoidal signal baseband signal.

Sinusoidal signal frequency estimation methods can be applied to periodic pulsar signal parameter estimation.

Pulsar characteristic frequency in the paper is defined as the number of pulsar- period changes during the period of observation.

According to Fourier transform:

$$
\begin{array}{r}
\hat{x}(k)=\sum_{n=0}^{N-1} x(n) w^{-n k} \\
x(n)=\frac{1}{N} \sum_{k=0}^{N-1} \hat{x}(k) w^{n k}
\end{array}
$$

The $x(n) \leftrightarrow \hat{x}(k)$ is the discrete Fourier transform pair and $w=e^{j \frac{2 \pi}{N}}$.

For the specific period, the pulsar signal can be given the estimated at $\mathrm{k}$ in the frequency spectrum of the signal.

$$
\hat{P}(k)=\left|\sum_{n=0}^{N-1} x(n) w^{-n k}\right|^{2}
$$

For the period $\mathrm{T}$ of the pulsar signal, there will be $f=\frac{1}{T}$ frequency $\mathrm{K}$. K can be detected at a peak of the spectrum.

The physical meaning of $\mathrm{K}$ is reflected in the number of pulsars periodic changes for the observation period.

If there is a peak, then the period $\mathrm{T}$ of the pulsar signal appears; if not, it means that there is no signal from the pulsar period.

We can have an example as follow.

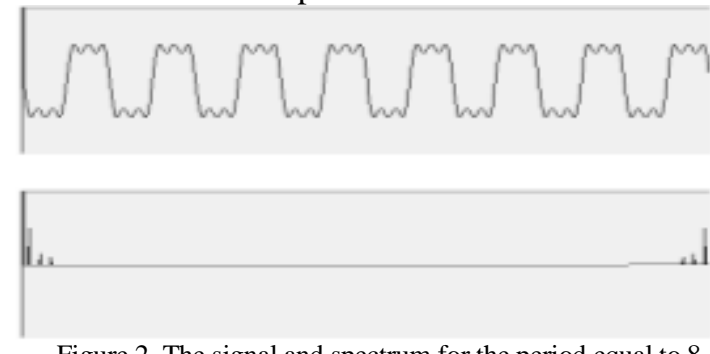

Figure 2. The signal and spectrum for the period equal to 8

$\mathrm{N}$ is 1024 points. In the observation range, the signal has changed 8 times .Corresponding spectrum peak appears at $\mathrm{k}=8$.

Since the signal is approximately a square wave, and therefore the 3rd harmonic and 5th harmonic frequencies also appeared at the peak.

Respectively, the amplitude of the 3rd and 5th harmonic is the amplitude of fundamental wave $1 / 3$ and $1 / 5$.

According to the theory of linear systems, the fundamental frequency component of the signal is retained in the delay information signal. The phase delay of the original signal is corresponding to that of the characteristic frequency signal

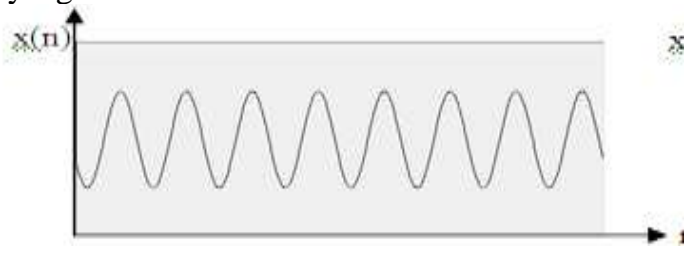

Figure 3. Relative delay is $\tau=10$

\section{B. pulsar signal preprocessing}

Let pulsar signal is $\boldsymbol{S}(\boldsymbol{t})$, due to the presence of noise, the sensor output is measured

$$
x(t)=s(t)+v(t)
$$

Assuming v (t) is Gaussian white noise, with the following probability distribution

$$
p(v)=\frac{1}{\sqrt{2 \pi} \sigma} e^{-\frac{v^{2}}{2 \sigma^{2}}}
$$

For a Gaussian white noise, the mathematical expectation of $\boldsymbol{x}(\boldsymbol{t})$ is

$$
E(x)=E(s+v)=s
$$

Variance is

$$
D(x)=E[x-E(x)]^{2}=\sigma^{2}
$$


Therefore, a good estimate can be obtained by averaging the measuring values of pulsar signal .For example; we use $M$ cycle's measurements of the pulsar signal values $x_{i}$ to estimate the pulsar signal $s$

$$
\hat{s}=\left(x_{0}+x_{1}+\cdots+x_{M-1}\right) / M=\bar{x}
$$

The root mean square of the signal is

$$
\begin{aligned}
& \sqrt{\bar{x}^{2}}=\sqrt{\left(\frac{1}{M} \sum_{i=0}^{M-1} x_{i}\right)^{2}}=\sqrt{\left(s+\frac{1}{M} \sum_{i=0}^{M-1} v_{i}\right)^{2}} \\
& =\sqrt{s^{2}+2 s \frac{1}{M} \sum_{i=0}^{M-1} v_{i}+\left(\frac{1}{M} \sum_{i=0}^{M-1} v_{i}\right)^{2}}
\end{aligned}
$$
results

As the $v(t)$ is Gaussian white noise, we can have the

$$
\begin{aligned}
& E\left(v_{i}\right)=0 \\
& E\left(v_{i} v_{j}\right)=\sigma^{2} \delta(i-j)
\end{aligned}
$$

So in (9), the second part is 0 , the third part could be simplified to the next value

$$
\begin{aligned}
\left(\frac{1}{M} \sum_{i=0}^{M-1} v_{i}\right)^{2} & =\frac{1}{M}\left(\frac{1}{M} \sum_{i=0}^{M-1} v_{i}^{2}\right)=\frac{\sigma^{2}}{M} \\
\sqrt{\bar{x}^{2}} & =\sqrt{s^{2}+\frac{1}{M} \sigma^{2}}
\end{aligned}
$$

SNR is defined as

$$
\frac{S}{N}=\frac{s}{\sigma}
$$

According to (12) we can obtain Signal-to-noise ratio of the pulsar using the average method

$$
\left(\frac{S}{N}\right)_{M}=\frac{s}{\frac{1}{\sqrt{M}} \sigma}=\sqrt{M} \frac{s}{\sigma}=\sqrt{M} \frac{S}{N}
$$

It shows after the signal-to-noise ratio has been improved $\sqrt{M}$ times after $\mathrm{M}$ times average processing .The average processing has the advantage of simplicity and easy operation. Average processing method is often used in many standard templates.

\section{III.}

\section{CRB THEORY}

As described above, a periodic signal can be decomposed into the sum of a series of sinusoidal signals, and the baseband signal can describe the basic characteristics of a periodic signal. The baseband signal is thus called the characteristic frequency signal. By using characteristic frequency signal method, the parameters estimation of the periodic signal in the amplitude, frequency and time delay change into the characteristics of the signal parameter estimation. Since the period of the pulsar signal is known, the characteristic frequency is also known. From the perspective of parameter estimation, the characteristic frequency estimation is accurate and unbiased.

For unbiased frequency estimator, Its best estimate of performance (such as minimum variance) can be compared with Cramer - Rao performance limit If the estimate is close to or equal to the variance Cramer - Rao limit, it can be considered that the estimator is optimal.

For the estimate of a single sinusoidal signal in Gaussian noise environments, Modern spectral estimation theory has proven the formula of Cramer - Rao limit is like

$$
\operatorname{var}(\hat{f}) \geq \frac{6 \sigma^{2}}{A^{2} N\left(N^{2}-1\right)(2 \pi)^{2}}
$$

$$
\begin{aligned}
& \operatorname{var}(\hat{A}) \geq \frac{\sigma^{2}}{2 N} \\
& \operatorname{var}(\hat{\phi}) \geq \frac{\sigma^{2}(N-1)}{A^{2} N(N+1)}
\end{aligned}
$$

Where $\sigma^{2}$ the variance of the Gaussian noise is, $A$ is the amplitude of sinusoidal signal, $\mathrm{N}$ is the length of the data sequence which is obtained by sampling the original signal.

It shows that Frequency estimation Cramer - Rao limit

$$
\frac{A^{2}}{\sigma^{2}}
$$

is inversely proportional to $\operatorname{SNR} \sigma^{2}$ and the cube of the length of the data appropriately selection of the data sequence length is a very important for parameter estimation As the pulsar signal cycle is known, the estimated frequency parameters can be considered to achieve Cramer - Rao limit

\section{TEST AND ANALYSIS}

This data selection is the part of the XTE satellite observation data. The data source is downloaded from the HEASARC website and the data format is FITS

\section{A. Pulsar Identificatio}

First we select the pulsar PSRJ0007 +7303 which has the period of $\mathrm{P}=315.8$ Sampling frequency is $633 \mathrm{~Hz}$.In the matlab, we draw the original signal just as Fig.4

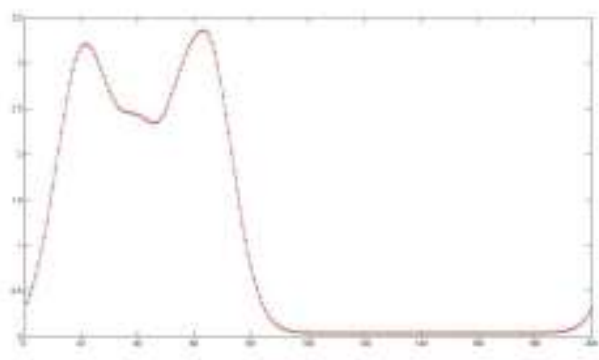

Figure 4 . PSRJ0007 +7303 time domain wave 


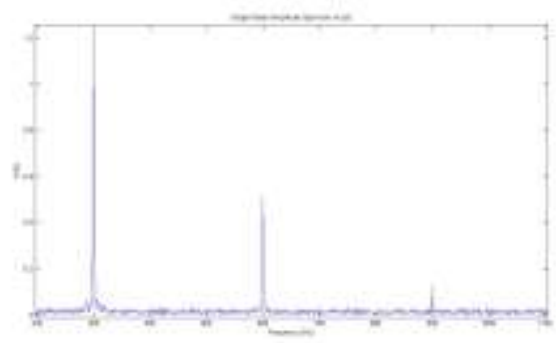

Figure 5. PSRJ0007 +7303 frequency domain spectrum

Then we plus a new pulsar PSRJ0023 +0923. And make recognition of these two mixed signals. The results are as follows. The algorithm can effectively identify these two pulsars

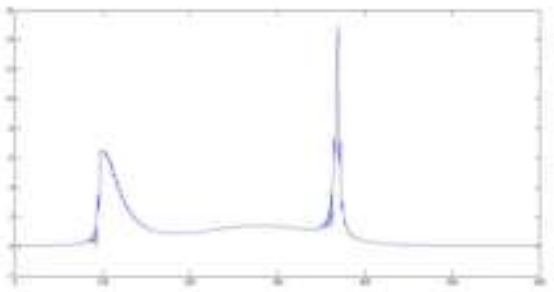

Figure 6 PSRJ0023 +0923 time domain wave

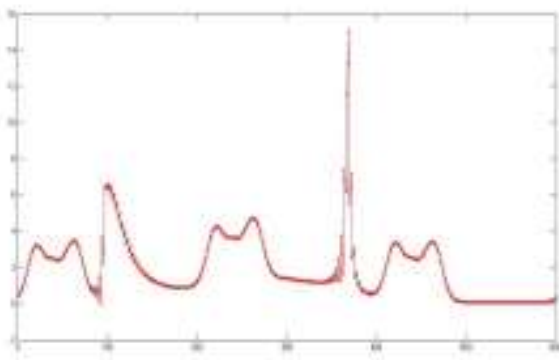

Figure7. PSRJ0007 +7303 plus PSRJ0023 +0923 time domain wave

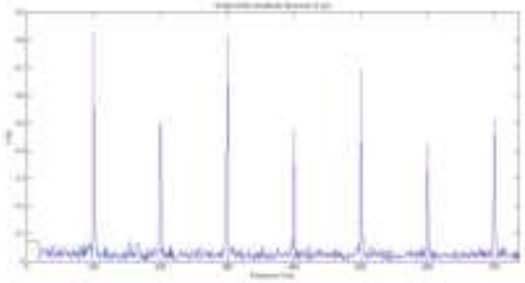

Figure 8. PSRJ0007 +7303 plus PSRJ0023 +0923 Frequency domain spectrum

\section{B. The estimation of phase}

Assuming a phase change of $\frac{\pi}{2}$ we operate 1000 times Monte Carlo simulation by matlab and make statistics of the results. The result figure is as follows.

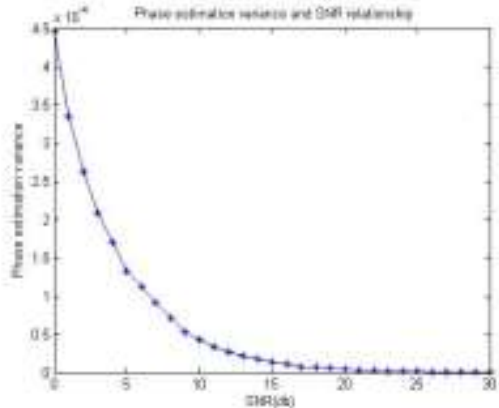

Figure9 the phase estimation variance and SNR relationship

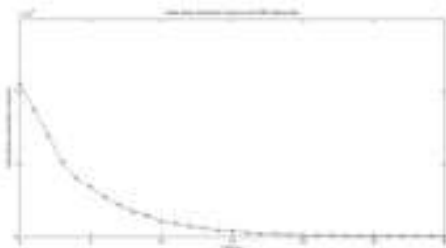

Figure10. the original phase variance and SNR relationship

\section{CONCLUSION}

(1)The paper selects pulsar PSRJ0007 +7303, plus Gaussian noise and uses the characteristic frequency identification method to achieve the recognition of single pulsars signal. Through the simulation of matlab ,the algorithm can achieve the single pulsar effective recognition

(2)On the basis of pulsars PSRJ0007 +7303 signal, $\mathrm{n}$ a new pulsar PSRJ0023 +0923, is joined in and the simulation is completed by matlab. The algorithm can achieve more stars pulse recognition effectively

(3)Phase change

The experiment choose pulsar PSRJ0007 +7303 . The number of FFT is $\mathrm{N}=50000$ and the SNR changes from 0 $\mathrm{db}$ to $30 \mathrm{db}$.The paper make statistics of the results from 1000 times Monte Carlo simulation .The statistics result shows the the pulsar characteristic frequency identification algorithm can measure the change of phase accurately. The variance is close to the best estimate bound- Cramer-Rao limit

\section{REFERENCES}

[1] Maron O, Kijak J, Kramer M, et al. Pulsar Spectra of Radio Emission[J]. Astronomy and Astrophysics, 2007,147:195-203.

[2] Morii M, Ktamoto S, Shibazaki N, et al. Suzaku Observation of the Anomalous X-Ray Pulsar[J]. Publications of The Astronomical Society of Japan, 2010, 62(5):1249-1259.

[3] Joseph H., Taylor JR. Millisecond Pulsars: Nature.s Most Stable Clocks. Proceedings of the IEEE. 1991, 79(7). 31. N

[4] J. Sala, A. Urruela, X. Villares, et al. Feasibility Study for a Spacecraft Navigation System Relying on Pulsar Timing Information. 2004

[5] Manchester R N, Hobbs G B, Teoh A, et al. The Australia Telescope National Facility Pulsar Catalogue[J]. The Astronomical Journal, 2008, 129:1993-2006.

[6] K.S.Wood G. Fritz. P. Hertz etc. The USA X-ray Timing Experiment American Physical Society, April Meeting, Jointly Sponsored with the High Energy Astrophysics Division (HEAD) of the American Astronomical Society April 20 - 23, 2002 Albuquerque Convention Center Albuquerque, New Mexico 
[7] A.A. Emadzadeh, J.L. Speyer A Study of Pulsar Signals Modeling and Time Delay Estimation for Relative Navigation of Spacecrafts ION64th Annual Meeting April23-25 2007, Cambridge, Massachusetts

[8] S. I. Sheikh D. J. Pines, P.S. Ray, K.S. Wood, M. N. Lovellette M T. Wolff Spacecraft navigation using X-ray Pulsars Journal of Guid Wood K S. The HEAO-1 X-ray Source Catalog[J] The Astrophysical Journal Supplement Series. 1984, 56:507-649.

[9] ance Control and Dynamics VOL 29 No.1 2006

[10] S. I. Sheikh, D. J. Pines, P.S. Ray, et al. The Use of X-ray Pulsars for Spacecraft Navigation. Advances in the Astronautical Sciences. $2005,199$.
[11] S. I. Sheikh. The Use of Variable Celestial X-Ray Sources for Spacecraft Navigation. Maryland University, 2005.

[12] Petre R.ROSAT:The Roentgen Satellite. 2004, March[OL] http://heasarc.gsfc.nasa.gov/docs/rosat/rosat.html.

[13] A.A. Emadzadeh, J.L. Speyer. A Study of Pulsar Signals Modeling and Time Delay Estimation for Relative Navigation of Spacecrafts. ION New Technology Meeting. San Diego, CA. 2008.

[14] Xie Qiang, Xu Luping, Zhang Hua, Luo Nan. Modeling of X-ray Pulsar Cumulation Profile and Signal Identification[J].Acta Physica Sinica, 2012, 61(11): 22432248. (in Chinese) 